\title{
Molecular mechanisms of TUG1 in the proliferation, apoptosis, migration and invasion of cancer cells (Review)
}

\author{
HUI ZHOU ${ }^{1}$, LINA SUN ${ }^{1}$ and FUSHENG WAN ${ }^{2}$ \\ ${ }^{1}$ The Second Clinical Medical College, Nanchang University; ${ }^{2}$ Department of Biochemistry and Molecular Biology, \\ Medical College of Nanchang University, Nanchang, Jiangxi 330006, P.R. China
}

Received January 10, 2019; Accepted August 14, 2019

DOI: $10.3892 / \mathrm{ol} .2019 .10848$

\begin{abstract}
Long non-coding RNAs (lncRNAs) are RNA sequences $>200$ nucleotides in length that have no protein-coding capacity. lncRNAs serve key roles in multiple biological processes, such as tumorigenesis and tumor progression. Taurine upregulated 1 (TUG1) is a novel lncRNA that has been associated with human cancer. TUG1 has attracted increasing attention in recent years and has been documented to be abnormally expressed in different types of cancer. Numerous studies indicate that TUG1 may be significantly associated with tumor development and cell metabolism by regulating cell proliferation, invasion, metastasis, apoptosis, differentiation and drug resistance. TUG1 exerts its function via recruiting specific RNA-binding proteins, promoting target gene expression, influencing tumor angiogenesis and by functioning as a competing endogenous RNA (ceRNA). An increasing number of studies have demonstrated that ceRNAs serve a role in cancer development. TUG1 is considered to be a biomarker or a novel therapeutic target for the diagnosis and prognosis of different cancer types. The present review focuses on recent developments in the major underlying molecular mechanisms of TUG1 in cancer, including its role in cell proliferation, apoptosis, migration, invasion and drug resistance. Also discussed in the present review is the current knowledge regarding the regulation of TUG1.
\end{abstract}

\section{Contents}

1. Introduction

2. Molecular mechanisms of TUG1 in cancer

3. TUG1 in cancer cell proliferation and apoptosis

4. TUG1 in cancer migration and invasion

Correspondence to: Dr Fusheng Wan, Department of Biochemistry and Molecular Biology, Medical College of Nanchang University, 461 Bayi Avenue, Nanchang, Jiangxi 330006, P.R. China E-mail: wanfs01@163.com

Key words: long non-coding RNA, taurine upregulated 1, cancer, proliferation, apoptosis, migration, invasion, drug resistance
5. TUG1 in cancer drug resistance

6. Regulation and dysregulation of TUG1

7. Discussion and perspectives

\section{Introduction}

The eukaryotic genome transcribes a diverse range of RNA molecules, from long protein-coding mRNAs to non-coding RNAs (ncRNAs) (1). Approximately $70 \%$ of the human genome encodes ncRNAs, while protein-coding genes account for $<2 \%$ of transcribed RNA (2-5). ncRNAs can be divided into short ncRNAs ( $<200$ nucleotides in length) and long ncRNAs (lncRNAs; >200 nucleotides in length) (2-4). It is generally thought that lncRNAs lack protein-coding capabilities and, therefore, they were originally regarded as inconsequential transcriptional noise (2). With continuous advances in sequencing technology and large-scale genome sequencing projects, lncRNAs have become a major focus of research. LncRNAs have been reported to serve extensive roles in various cellular and physiological processes, such as chromatin dynamics, and transcriptional and post-transcriptional processes (6-8). Numerous studies have demonstrated that dysregulation of lncRNAs may be involved in the pathological process of several human diseases, including cancer $(9,10)$, cardiovascular disease (11) and diseases of the central nervous system (12). Through epigenetic modifications, chromatin remodeling and microRNA (miRNA) sponging, lncRNAs modulate cell proliferation, apoptosis, migration and invasion (13-15). Due to the tissue-specific expression and high stability of lncRNAs (which allows them to be detected in body fluids), they may be used as biomarkers for the effective diagnosis, prognosis and monitoring of disease progression (16).

Taurine up-regulated 1 (TUG1) is a 7,598-nucleotide lncRNA sequence localized to chromosome 22q12.2 and was originally identified in a genomic screen of taurine-treated mouse retinal cells $(17,18)$. TUG1 has been demonstrated to serve crucial regulatory roles in various cancer-associated biological processes and may provide a putative novel treatment paradigm in cancer therapy. The results of two previous meta-analyses have indicated that increased expression of TUG1 is an unfavorable predictor of survival in human cancer, and that TUG1 is closely associated with increased tumor size, advanced pathological stage and distant metastasis $(19,20)$. In 
addition, TUG1 is ubiquitously expressed and has been implicated in different cancer types. TUG1 has been demonstrated to be upregulated in renal cell carcinoma (21), ovarian cancer (22), bladder urothelial carcinoma (23), osteosarcoma (24), oral squamous cell carcinoma (25), esophageal squamous cell carcinoma (26), hepatocellular carcinoma (HCC) (27), intrahepatic cholangiocarcinoma (28), glioma (29), cervical cancer (30), endometrial cancer (31), pancreatic cancer (32), breast cancer (33), bladder cancer (34), colorectal cancer (CRC) (35), small cell lung cancer (SCLC) (36), melanoma (37), thyroid cancer (38), gallbladder carcinoma (39) and gastric cancer (GC) (40). By contrast, TUG1 expression has been observed to be downregulated in non-SCLC (NSCLC) (41), triple-negative breast cancer (TNBC) (42) and glioma (43). In addition, previous studies have reported that aberrant expression of TUG1 may affect gene expression through diverse mechanisms, which affect various biological processes, including cell proliferation, invasion, apoptosis, differentiation, migration, drug resistance, radiation resistance, angiogenesis, mitochondrial bioenergetics, epithelial-mesenchymal transition (EMT) and blood tumor barrier permeability regulation (44-49). By conducting an extensive search of the relevant literature, this review summarizes the key findings of previous studies and elaborates on the cellular functions and mechanisms of TUG1 regulation, as well as its role in human cancer, particularly focusing on its role in cell proliferation, invasion, apoptosis, migration and drug resistance.

\section{Molecular mechanisms of TUG1 in cancer}

Accumulating evidence suggests that the molecular function of IncRNAs is important in epigenetic regulation and that their molecular mechanisms may be diverse $(44,50)$. Recent studies have suggested that TUG1 is involved in gene regulation through a variety of mechanisms; primarily by functioning as a miRNA sponge and via interacting with polycomb repressive complex 2 (PRC2) $(41,51)$. TUG1 functions as a competing endogenous RNA (ceRNA) to target miRNAs, thereby inhibiting their biological functions $(28,29,31,37,42)$. This leads to changes in the expression level of downstream target genes. Khalil et al (51) confirmed that TUG1 recruits and binds with PRC2. PRC2 possesses methyltransferase activity and consists of enhancer of zeste homologue 2 (EZH2), suppressor of zeste 12 protein homolog (SUZ12), embryonic ectoderm development (EED) and retinoblastoma-associated protein 46/48. This complex catalyzes the di- and tri-methylation of lysine residue 27 on histone $3(52,53)$. The binding of TUG1 to PRC2 directs the concomitant genomic DNA to polycomb bodies leading to epigenetic silencing. The various molecular mechanisms of TUG1 are summarized in the following text.

\section{TUG1 in cancer cell proliferation and apoptosis}

It has been demonstrated that TUG1 is capable of promoting both the proliferation and apoptosis of cells $(18,21,24,26,30-34)$. Furthermore, TUG1 can exert the same biological function through the regulation of different target genes in different cell types $(27,28,31,36-39)$.

Investigating the interactions between lncRNAs and miRNAs is a popular focus of current ncRNA research (54).
As aforementioned, TUG1 exerts its function in cancer cell proliferation and apoptosis by serving as an miRNA sponge. In papillary thyroid cancer cells, TUG1 functions as a ceRNA to sequester miR-145, which in turn leads to an increase in the expression of the miR-145 target, zinc finger E-box binding homeobox 1 (ZEB1). Therefore, TUG1 mediates increased cell proliferation via regulating the miR-145/ZEB1 signaling pathway (38). In cervical cancer, TUG1 exerts an oncogenic role by binding to miR-138-5p and resulting in its neutralization. Sirtuin 1 (SIRT1) competes with miR-138-5p and, thus, is upregulated by TUG1. Highly expressed SIRT1 promotes the expression of c-myc, $\beta$-catenin and cyclin D1, and inhibits the expression of E-cadherin. This leads to activation of the Wnt/ $\beta$-catenin signaling pathway, and subsequent inhibition of apoptosis and promotion of cervical cancer cell proliferation and invasion (55).

A previous study reported that TUG1 serves an oncogenic role in human malignant melanoma via upregulating astrocyte elevated gene-1 (AEG1) expression by sponging miR-129-5p (37). AEG1 serves an important role in tumorigenesis via the phosphatidylinositol-4,5-bisphosphate 3-kinase (PI3K)/AKT (56) and Wnt signaling pathways (57). Activation of the TUG1/miR-129-5p/AEG-1 axis in malignant melanoma cells leads to the inhibition of proliferation and increased apoptosis (37). Suppression of TUG1 inhibits the expression of B-cell lymphoma-2 (Bcl-2), matrix metallopeptidase-9 and cyclin D1, and increases cleaved caspase 3 levels, via modulating the expression of AEG1 in melanoma cells. As a consequence, overexpression of TUG1 leads to tumorigenesis. Therefore, suppression of TUG1 expression may inhibit tumorigenesis and the proliferation of malignant human melanoma cells. Yan et al (58) reported that miR-219 suppresses the proliferation of oral squamous cell carcinoma cells by regulating the expression of formin-like 2 (FMNL2). Furthermore, it was demonstrated that TUG1 sponges miR-219 to regulate its expression in oral squamous cell carcinoma. In addition, Zhao and Ren (59) indicated that TUG1 promotes cell proliferation and inhibits apoptosis of breast cancer cells by functioning as an endogenous sponge of miR-9, thus affecting the expression of the miR-9 target gene, methylenetetrahydrofolate dehydrogenase ( $\mathrm{NADP}^{+}$-dependent) 2 . In gallbladder carcinoma, TUG1 increases cell proliferation by negatively regulating miR-300 (39); however, the downstream target gene of miR-300 remains unclear. miRNA-26a binding sites have been identified in the TUG1 sequence, and a negative correlation between TUG1 and miR-26a expression has been observed in prostate cancer (60). Therefore, the mechanisms underlying TUG1-mediated cell proliferation and apoptosis may be based, in part, on its negative regulation of miR-26a. In addition, TUG1 and miR-382 have been demonstrated to negatively regulate each other. The TUG1/miR-382/EZH2 signaling pathway has been identified as a ceRNA regulatory network involved in the proliferation of pancreatic cancer cells (61).

TUG1 has been reported to sequester miR-382 and downregulate its expression in pancreatic cancer (61). Similarly, TUG1 has been observed to interact with additional miRNAs, including miR-29c, miR-142 and miR-145 in bladder cancer (62-64). EZH2, a downstream target gene of miR-382, miR-142 and miR-145, is positively regulated 
by TUG1 $(61,63,64)$. Overexpression of TUG1 promotes the proliferation and induces the apoptosis of HCC cells. Furthermore, silencing of TUG1 reduced the expression of the sonic hedgehog protein, known to be associated with the Hedgehog (Hh) signaling pathway, and led to an increase in the expression of miR-132 in hepatocellular carcinoma cells (65). As reported previously, overexpression of miR-132 reduces cell proliferation and promotes apoptosis. The Hh gene was initially identified in Drosophila in 1980, and Hh has been implicated in the classical signaling pathway activated during embryonic development and cell differentiation (66). Considering these previous observations, it is thought that TUG1 may function as an oncogene and induce tumorigenesis by regulating the Hh signaling pathway. In addition, TUG upregulates the level of Janus kinase 2 epigenetically through binding to miR-144, thus promoting HCC cell proliferation (67).

In addition to the indirect regulation of ceRNAs, studies published to date have demonstrated that high TUG1 expression in osteosarcoma results in sponging 9 miRNAs, including miR-212-3p, miR-132-3p, miR-144-3p, miR-153, miR-9-5p and miR-219a-5p, which affects cell proliferation and apoptosis (68-73). Xie et al (68) demonstrated that TUG1 upregulated forkhead box A1 (FOXA1) expression and FOXA1-mediated cell proliferation and apoptosis by functioning as a ceRNA through miR-212-3p. In addition, TUG1 was observed to affect the expression of sex-determining region Y-box 4 by functioning as a ceRNA of miR-132-3p (69). TUG1 shares miR-144-3p-response elements with EZH2, which prevents it from undergoing miR-144-3p-mediated degradation (70). Furthermore it has been shown that TUG1 contributes to osteosarcoma tumorigenesis by sponging miR-153 (71). Mechanistically, TUG1 binds to miR-9-5p and thereby inhibits its activity. Concordantly, TUG1 upregulates the miR-9-5p downstream target gene, POU class 2 homeobox 1, which is involved in cell proliferation, colony formation, cell cycle arrest and apoptosis (72). A recent study has demonstrated that high TUG1 expression in osteosarcoma leads to the downregulation of miR-219a-5p expression via an endogenous sponge adsorption mechanism. This leads to further upregulation of PI3KCA and activation of the AKT signaling pathway, which promotes cell proliferation (73).

A major role of TUG1 is regulating gene expression and influencing cell proliferation via binding to PRC2. Modulation of TUG1 alters the level of multiple genes in various cancer cells; the majority of which are PRC2-dependent. A diverse number of genes are targeted by TUG1-induced PRC2, including CUGBP Elav-like family member 1 (CELF1), homeobox B7 (HOXB7), LIM domain kinase 2b (LIMK2b), p57, Kruppel-like factor 2 (KLF2) and Bax; a number of these are positive regulators of cell proliferation and apoptosis $(27,36,41,74-78)$. Lin et al (41) demonstrated that the expression levels of TUG1 are prominently downregulated in NSCLC tumors. In addition, knockdown of TUG1 significantly increases the proliferation of NSCLC cells. TUG1 affects NSCLC cell proliferation by negatively regulating CELF1. Of particular note is that chromatin immunoprecipitation assays have confirmed that EZH2/EED bind to the promoter region of CELF1 at 992 bp upstream of the transcription start site. Consequently, TUG1 binds with PRC2 and occupies the promoter region of CELF1. An additional study reported that
TUG1 is required to activate PRC2 by occupying its binding site on HOXB7 (a known oncogene), thereby epigenetically modulating its expression and promoting NSCLC cell proliferation $(74,75)$. In SCLC, TUG1 downregulates LIMK2b expression by binding with EZH2 to promote SCLC cell proliferation (36). In addition, TUG1 recruits EZH2 to target gene promoters and epigenetically represses the expression of cyclin-dependent protein kinase inhibitors, including p15, p16, p21, p27 and p57 in GC. This leads to TUG1-mediated alterations in the proliferation of GC cells and cell cycle progression (76). Ultimately, the results of these studies indicate that TUG1 may function as a regulator of cell proliferation. In addition, knockdown of TUG1 in a HCC cell line, Hep3B, led to inhibition of cell proliferation and induction of cell apoptosis in vitro (27). TUG1 recruits PRC2 to the KLF2 gene locus, which leads to the direct binding of EZH2 to its promoter region and inactivation of KLF2 expression. The low expression levels of KLF2 (a known tumor suppressor gene) results in reduced cell apoptosis and permits increased proliferation of HCC cells (27,77). TUG1 regulates cancer cell apoptosis in lung adenocarcinoma via inhibition of Bax, which is a pro-apoptotic protein that interacts with EZH2 (78).

In ovarian epithelial cancer cells, TUG1 has been reported to inhibit apoptosis and promote cell proliferation by upregulating the expression of aurora kinase A, which has been shown to be implicated in the carcinogenic processes of multiple human cancer types (79-81). In addition, knockdown of TUG1 in oral squamous cell carcinoma cells leads to decreased proliferation in vitro (25). Reverse transcription-quantitative PCR and western blotting results indicated that knockdown of TUG1 leads to downregulation of $\mathrm{Wnt} / \beta$-catenin signaling-associated genes, such as $\beta$-catenin, cyclin D1 and c-myc (25). In general, TUG1 promotes cell growth and proliferation via targeting the Wnt/ $\beta$-catenin signaling pathway.

Recent studies have proposed that TUG1 exerts anti-apoptotic effects in CRC (82), bladder cancer (23), ovarian cancer (22), oral squamous cell carcinoma (25) and cervical cancer (30) cells by regulating the expression of apoptosis-associated mediators, including caspase 3, caspase 9 and Bcl-2. Yin et al (83) demonstrated that the level of TUG1 expression in pancreatic tissues is significantly higher than that in other organ tissues.

\section{TUG1 in cancer migration and invasion}

It has been reported that EMT serves a significant role in mediating migration and invasion (84). During EMT, the expression of epithelial cell markers, including E-cadherin, cytokeratins and claudins, is downregulated. By contrast, the expression of vimentin, $\mathrm{N}$-cadherin, fibronectin and mucin 1 , which function as mesenchymal markers, is upregulated (85). Recently, an association between TUG1 and EMT was identified. When TUG1 expression is knocked down in ovarian cancer cells, E-cadherin expression is high, while vimentin and $\mathrm{N}$-cadherin levels are downregulated. Simultaneously, the proliferation and metastasis of ovarian cancer cell lines are markedly inhibited. These results suggest that TUG1 may participate in modulating cell migration and invasion via EMT (22). Similarly, in bladder and cervical cancer, TUG1 was demonstrated to promote cell invasion by inducing EMT $(30,34)$. In addition, overexpression 
of TUG1 promoted the migration, invasion and EMT of thyroid cancer cell lines, and these effects were primarily dependent on the TUG1-mediated regulation of the miR-145/ZEB1 signaling pathway (38). Furthermore, it was demonstrated that knockdown of TUG1 markedly inhibited the invasion and migration capabilities of prostate cancer cells (61). Mechanistically, silencing of TUG1 promotes changes in cell morphology from a mesenchymal to an epithelial phenotype. TUG1 facilitates pancreatic cancer cell invasion by stimulating ZEB2-mediated EMT (61). In addition, TUG1 has been reported to accelerate EMT by promoting the expression of KIAA1199 (also known as cell migration-inducing hyaluronidase 1) by reducing miR-600 expression (86). It was demonstrated that high levels of miR-600 inhibited CRC migration, invasion and the expression of EMT-associated proteins in vitro. However, KIAA1199 was indirectly upregulated by TUG1 (86).

Following confirmation that miR-219a-5p targets TUG1 using a luciferase assay, it was demonstrated that TUG1 regulates the expression of PIK3CA and activates the AKT signaling pathway to promote the migration and invasion of osteosarcoma cells (87). In addition, TUG1 was observed to downregulate the expression of miR-335-5p in osteosarcoma via an endogenous sponge adsorption mechanism, which led to further upregulation of $\rho$-associated coiled-coil containing protein kinase 1 and increased cell migration and invasion (73). A recent study reported that TUG1 induces the invasion of oral squamous cell carcinoma cells via repression of miR-219 or regulation of FMNL2 (58). In addition, Zhao et al (61) demonstrated that TUG1 promotes the migration and EMT of pancreatic cancer cells via downregulation of miR-382 and regulation of its target, EZH2 (61). Furthermore, miR-144 has been demonstrated to bind directly with TUG1. Recent studies have investigated whether TUG1 may function as a ceRNA and bind to miRNA response elements in miR-144, thus inhibiting its expression in GC and HCC $(67,88)$. Together, these studies provide evidence indicating a significant correlation between increased TUG1 expression and invasion. Ji et al (88) demonstrated that TUG1 is markedly upregulated in GC tissues and that silencing of TUG1 leads to the inhibition of c-Met and the inhibition of invasion capabilities of SGC-7901 cells. In HCC, TUG1 competes with miR-144 for binding to the 3'-untranslated region of Janus kinase 2 (JAK2), thereby activating the JAK2/signal transducer and activator of transcription 3 signaling pathway and increasing cell migration in vitro and in vivo (67). He et al (89) demonstrated that high TUG1 levels were positively associated with cell invasion in HCC. TUG1 directly interacts with miR-142-3p. In addition, miR-142-3p suppresses HCC cell invasion and metastasis by targeting ZEB1. Silencing of TUG1 consequently reduces the expression levels of ZEB1 and EMT-associated proteins. Moreover, TUG1 acts as an oncogene in HCC progression by binding with miR-142-3p and abrogating its tumor-suppressive function. Furthermore, it has been reported that the TUG1/PRC2/p21/miR-455-3p/AMPK $\beta 2$ axis regulates hexokinase 2 (HK2) to mediate HCC cell invasion (90). TUG1 downregulates p21 expression via upregulation of EED expression, which leads to reduced p21-E2F1 interaction and enhanced miR-455-3p expression. The expression of AMPK $\beta 2$ is inhibited by upregulation of miR-455-3p and thereby HK2 expression is increased.

\section{TUG1 in cancer drug resistance}

Drug resistance is one of the most important reasons for therapeutic failure in patients with cancer and is a persistent issue that requires continued investigation. Tang et al (42) demonstrated that the expression of miR-197 is elevated in human TNBC tumor tissues and cell lines (MDA-MB-231 and BT549), while the levels of TUG1 and nemo-like kinase (NLK) are decreased. miR-197 confers cisplatin resistance in TNBC by inhibiting NLK. However, TUG1 functions as an endogenous sponge of miR-9-5p. Increased expression of TUG1 sensitizes TNBC cells to very low concentrations of cisplatin. Therefore, the TUG1/miR-197/NLK signaling pathway is likely to be a novel and promising therapeutic target for patients with TNBC.

TUG1 has been implicated in the resistance of human CRC cells to methotrexate (MTX) via the miR-186/cytoplasmic polyadenylation element binding protein 2 (CPEB2) axis (91). MTT assays have demonstrated that miR-186 re-sensitizes CRC cells to MTX. By contrast, CPEB2 enhances the resistance of CRC cells to MTX. TUG1 functions as a ceRNA to upregulate CPEB2 by sponging miR-186. Overexpression of TUG1 significantly enhances MTX resistance in CRC cells, which suggests that TUG1 may present a putative therapeutic target in CRC.

\section{Regulation and dysregulation of TUG1}

The expression level of TUG1 and the association between expression and survival probability in several common tumors were searched using The Cancer Genome Atlas database, including HCC, melanoma, osteosarcoma, renal cell carcinoma, bladder cancer, CRC, GC and triple negative breast cancer. The figures of the statistical results were obtained from this database (Figs. 1 and 2). According to The Cancer Genome Atlas database, there is a marked difference in the expression of TUG1 between normal and tumor tissues (Fig. 1). TUG1 serves both oncogenic and tumor suppressive functions depending on the type of cancer. As an oncogene, aberrant upregulation of this lncRNA in different cancer types compared with their noncancerous counterparts has been observed in HCC, melanoma, osteosarcoma, renal cell carcinoma, bladder cancer, CRC and GC. By contrast, other reports have observed downregulation of TUG1 in TNBC. However, it has been demonstrated that high TUG1 expression may indicate a poor prognosis in the majority of cancer types (Fig. 2).

A recent bioinformatics study demonstrated that the TUG1 promoter region harbors five SP1 binding sites, and it has been reported that SP1 directly binds to the TUG1 promoter region to upregulate TUG1 expression (27). In addition, Zhang et al (74) demonstrated that TUG1 is a direct transcriptional target of p53. p53 binds to its putative response element in the promoter region of TUG1. The p53 tumor suppressor gene is associated with the occurrence and development of various cancer types, such as breast cancer (92), bladder cancer (93), liver cancer (94) and glioma (95). It is possible that TUG1 may be closely associated with p53 and cancer; however, further research to verify this hypothesis is required. A previous study involving RNA-sequence and lncRNA microarray analyses has demonstrated that TUG1 is commonly downregulated in 

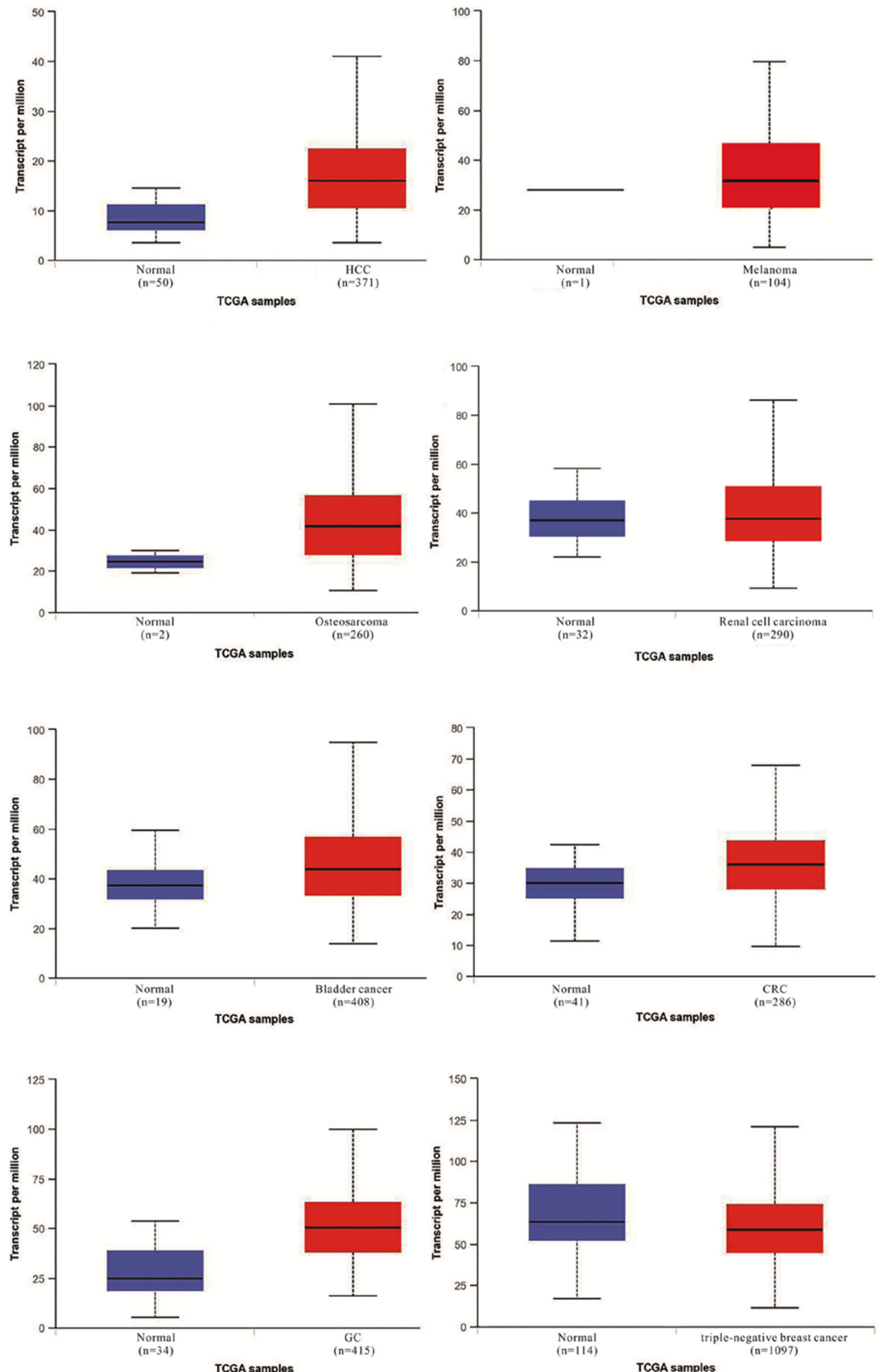

Figure 1. Differences in the expression of TUG1 between normal and tumor tissues. TUG1, taurine upregulated 1; HCC, hepatocellular carcinoma; CRC, colorectal cancer; GC, gastric cancer; TCGA, The Cancer Genome Atlas. 

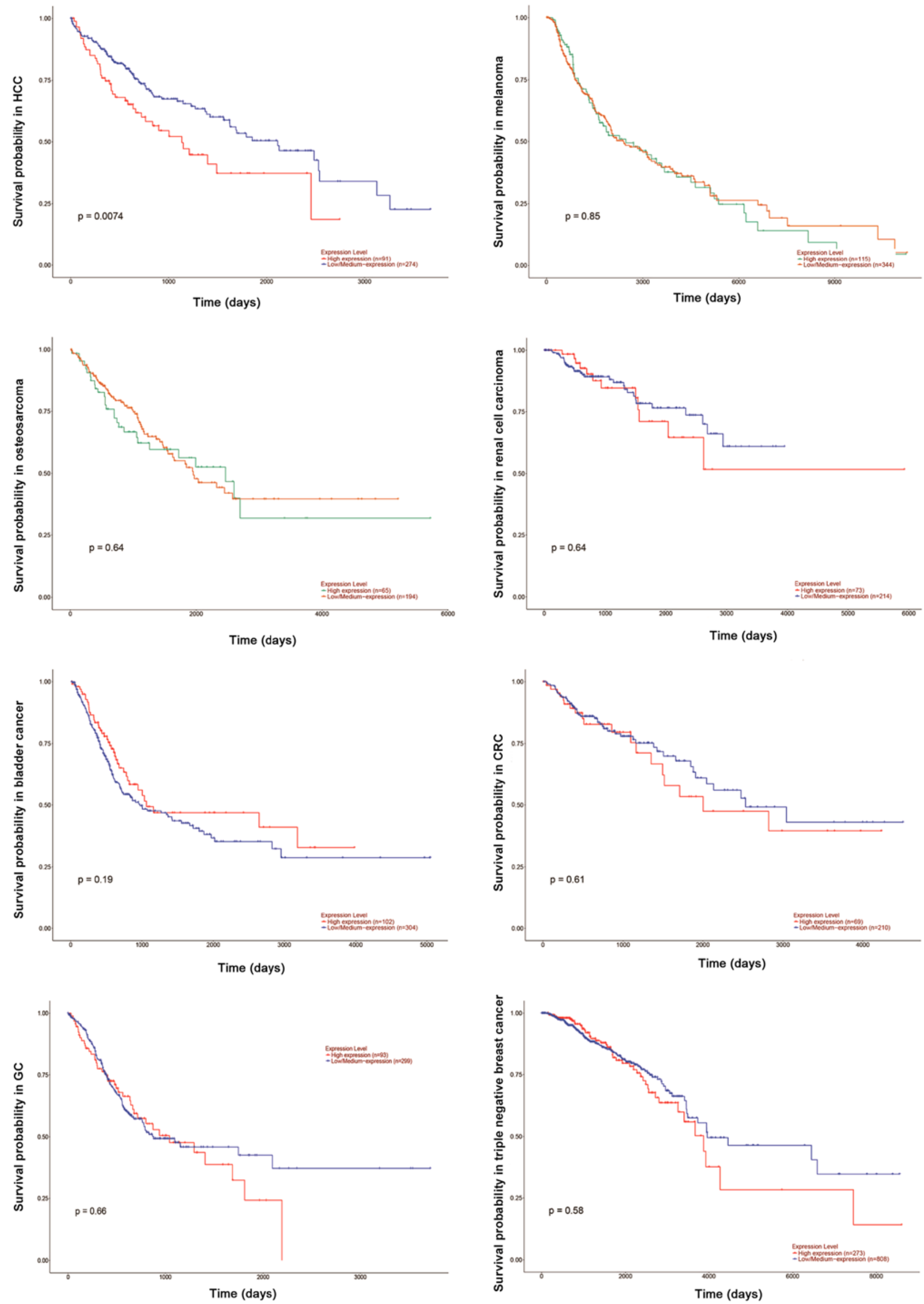

Figure 2. Association between TUG1 expression and survival according to The Cancer Genome Atlas dataset. TUG1, taurine upregulated 1; HCC, hepatocellular carcinoma; CRC, colorectal cancer; GC, gastric cancer. 


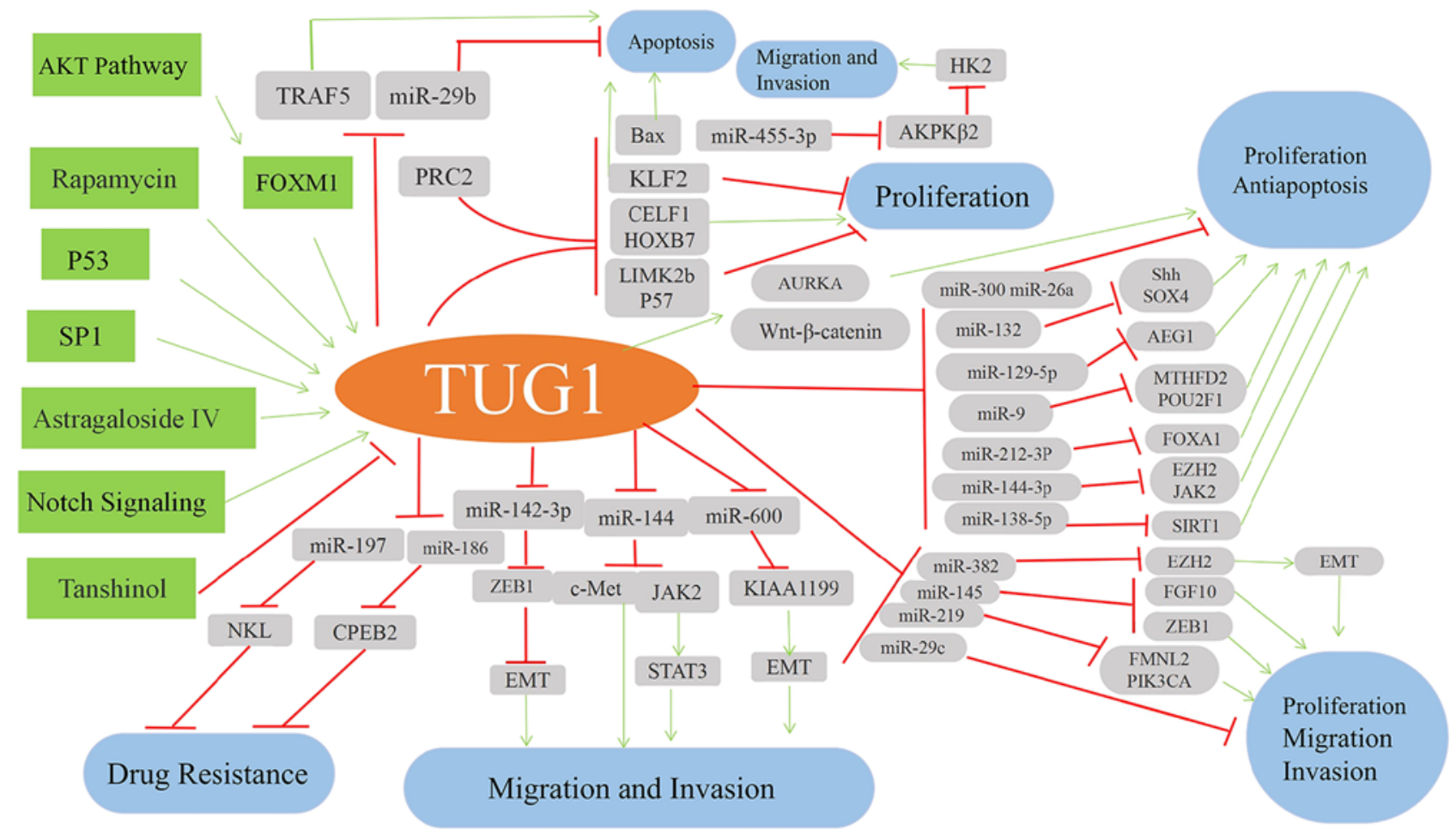

Figure 3. Schematic illustration of TUG1 regulatory mechanisms. TRAF5, TNF receptor-associated factor 5; FOXM1, forkhead box M1; PRC2, polycomb repressive complex 2; CELF1, CUGBP Elav-like family member 1; HOXB7, homeobox B7; LIMK2b, LIM domain kinase 2b; KLF2, Kruppel-like factor 2; AURKA, aurora kinase A; HK, hexokinase 2; AEG1, astrocyte elevated gene-1; FOXA1, forkhead box A1; EZH2, enhancer of zeste homologue 2; JAK2, Janus kinase 2; SIRT1, Sirtuin 1; ZEB1, zinc finger E-box binding homeobox 1; CPEB2, cytoplasmic polyadenylation element binding protein 2; KIAA1199, migration inducing hyaluronidase 1; EMT, epithelial-mesenchymal transition.

glioma following inhibition of Notch signaling. Notch1 was identified to bind with recombination signal binding protein for immunoglobulin $\kappa \mathrm{J}$ region motifs located upstream of the transcription start site of TUG1. TUG1 expression is induced by Notch signaling (46).

TUG1 has been reported to be induced by forkhead box M1 (FOXM1) in osteosarcoma cells (73). Genome-wide gene expression profiling of human solid tumors has demonstrated that FOXM1 is one of the most commonly upregulated genes across different cancer types $(96,97)$. AKT activates FOXM1 by inducing the phosphorylation of FOXO3 for protein degradation in osteosarcoma cells. TUG1 and FOXM1 are concurrently highly expressed in human osteosarcoma. Consistent with these observations, increased FOXM1 expression was demonstrated to activate the TUG1 promoter in a dose-dependent manner. In addition, induction of TUG1 by FOXM1 is diminished when FOXM1 expression levels are silenced by small interfering RNA (siRNA). These observations suggest that increased FOXM1 expression by AKT may upregulate the expression level of TUG1 via direct interaction with the TUG1 promoter in osteosarcoma cells.

\section{Discussion and perspectives}

lncRNAs, which are non-protein-coding RNA sequences, serve important roles in multiple cellular processes. TUG1, as a novel lncRNA, has been demonstrated to be abnormally expressed in various human cancer types, and its dysregulation is closely associated with carcinogenesis and disease progression. Emerging evidence suggests that TUG1 may be a vital mediator of cell proliferation, apoptosis, migration, invasion and drug resistance. Further study of TUG1, which is considered to function as a gene regulator, may aid in addressing disease etiology. From a molecular perspective, cancer is a genetic disease that develops due to the aberrant expression and function of tumor suppressor and oncogenic genes. TUG1 functions as an oncogene or tumor suppressor depending on the type of cancer. As such, silencing or upregulating TUG1 may inhibit the proliferation, migration and invasion of cancer cells and increase cell apoptosis. The present review summarizes the mechanisms of TUG1 in mediating these biological processes and discusses the regulation of TUG1 (Fig. 3).

Notwithstanding progress in the field of lncRNA research in recent years, studies investigating the role of TUG1 in cancer are still at a very early stage. For instance, Li et al $(43,98)$ has demonstrated that TUG1 is significantly reduced in U251 and SHG-44 glioma cells. This has provided a novel insight for the potential treatment of patients with glioma by overexpressing TUG1. By contrast, other studies have reported that TUG1 is upregulated in glioma tissues and cell lines $(29,45)$ and therefore functions as a putative oncogene. Therefore, the role of TUG1 in glioma is contradictory and requires further investigation.

In conclusion, the regulatory network of TUG1 in the majority of biological processes varies considerably. However, TUG1 appears to mediate these processes primarily by binding with PCR 2 to silence downstream target genes and by sponging miRNAs to promote the expression of target genes. In theory, 
TUG1 activity may be inhibited using numerous strategies. One approach may be to inhibit molecular interactions using small molecule inhibitors that block specific binding sites. Alternatively, TUG1 may be silenced using specific siRNAs. Investigation of TUG1 signaling pathways in cancer cells may uncover numerous novel therapeutic approaches for cancer treatment in the future. Therefore, it is essential for future research to focus on understanding the underlying molecular mechanisms of TUG1.

\section{Acknowledgements}

Not applicable.

\section{Funding}

The present study was supported in part by the grants from the National Natural Science Foundation of China (grant no. 81360032) and the Jiangxi Natural Science Foundation (grant no. 20161BAB205206).

\section{Availability of data and materials}

The datasets generated during the current study are available in the Cancer Genome Atlas repository, (https://portal.gdc. cancer.gov).

\section{Authors' contributions}

$\mathrm{HZ}$ and LS conceived and designed the review. HZ drafted the manuscript. LS participated in the manuscript's revision and provided suggestions for important content. FW proposed concepts, revised the article and obtained funding. All the authors approved the final version of manuscript.

\section{Ethics approval and consent to participate}

Not applicable.

\section{Patient consent for publication}

Not applicable.

\section{Competing interests}

The authors declare that they have no competing interests.

\section{References}

1. Reik W: Evolution and functions of long noncoding RNAs. Cell 136: 629-641, 2009.

2. Wahlestedt C: Targeting long non-coding RNA to therapeutically upregulate gene expression. Nat Rev Drug Discov 12: 433-446, 2013.

3. Lee JT: Epigenetic regulation by long noncoding RNAs. Science 338: 1435-1439, 2012.

4. Ling H, Fabbri M and Calin GA: MicroRNAs and other non-coding RNAs as targets for anticancer drug development. Nat Rev Drug Discov 12: 847-865, 2013.

5. Du Z, Sun T, Hacisuleyman E, Fei T, Wang X, Brown M, Rinn JL, Lee MG, Chen Y, Kantoff PW and Liu XS: Integrative analyses reveal a long noncoding RNA-mediated sponge regulatory network in prostate cancer. Nat Commun 7: 10982, 2016.
6. Bhan A and Mandal SS: LongNoncoding RNAs: Emerging stars in gene regulation, epigenetics and human disease. ChemMedChem 9: 1932-1956, 2015.

7. Heo JB, Lee YS and Sung S: Epigenetic regulation by long noncoding RNAs in plants. Chromosome Res 21: 685-693, 2013.

8. Mercer TR, Dinger ME and Mattick JS: Long non-coding RNAs: Insights into functions. Nat Rev Genet 10: 155-159, 2009.

9. Wapinski $\mathrm{O}$ and Chang HY: Long noncoding RNAs and human disease. Trends Cell Biol 21: 354-361, 2011.

10. Yang G, Lu X and Yuan L: LncRNA: A link between RNA and cancer. Biochim Biophys Acta 1839: 1097-1109, 2014.

11. Schonrock N, Harvey RP and Mattick JS: Long noncoding RNAs in cardiac development and pathophysiology. Circ Res 111: 1349-1362, 2012.

12. Carrieri C, Forrest A, Santoro C, Persichetti F, Carninci P, Zucchelli S and Gustincich S: Expression analysis of the long non-coding RNA antisense to Uchl1 (AS Uchl1) during dopaminergic cells' differentiation in vitro and in neurochemical models of Parkinson's disease. Front Cell Neurosci 9: 114, 2015.

13. Ulitsky I and Bartel DP: lincRNAs: Genomics, evolution and mechanisms. Cell 154: 26-46, 2013.

14. Rinn JL: lncRNAs: Linking RNA to chromatin. Cold Spring Harb Perspect Biol 6: pii: a018614, 2014.

15. Wilusz JE: Long noncoding RNAs: Re-writing dogmas of RNA processing and stability. Biochim Biophys Acta 1859: 128-138, 2016.

16. Kunej T, Obsteter J, Pogacar Z, Horvat S and Calin GA: The decalog of long non-coding RNA involvement in cancer diagnosis and monitoring. Crit Rev Clin Lab Sci 51: 344-357, 2014.

17. Young TL, Matsuda T and Cepko CL: The noncoding RNA Taurine Upregulated gene 1 is required for differentiation of the murine retina. Curr Biol 15: 501-512, 2005.

18. Zhang Q, Geng PL, Y in P, Wang XL, Jia JP and Yao J: Down-regulation of long non-coding RNA TUG1 inhibits osteosarcoma cell proliferation and promotes apoptosis. Asian Pac J Cancer Prev 14: 2311-2315, 2013.

19. Zhou Y, Lu Y, Li R, Yan N, Li X and Dai T: Prognostic role of long non-coding RNA TUG1 expression in various cancers: A meta-analysis. Oncotarget 8: 100499-100507, 2017.

20. Li N, Shi K, Kang X and Li W: Prognostic value of long non-coding RNA TUG1 in various tumors. Oncotarget 8: 65659-65667, 2017.

21. Zhang M, Lu W, Huang Y, Shi J, Wu X, Zhang X, Jiang R, Cai Z and $\mathrm{Wu} \mathrm{S}$ : Downregulation of the long noncoding RNA TUG1 inhibits the proliferation, migration, invasion and promotes apoptosis of renal cell carcinoma. J Mol Histol 47: 421-428, 2016.

22. Kuang D, Zhang X, Hua S, Dong W and Li Z: Long non-coding RNA TUG1 regulates ovarian cancer proliferation and metastasis via affecting epithelial-mesenchymal transition. Exp Mol Pathol 101: 267-273, 2016.

23. Han Y, Liu Y, Gui Y, Gui Y and Cai Z: Long intergenic non-coding RNA TUG1 is overexpressed in urothelial carcinoma of the bladder. J Surg Oncol 107: 555-559, 2013.

24. Yun-Bo F, Xiao-Po L, Xiao-Li L, Guo-Long C, Pei Z and Fa-Ming T: LncRNA TUG1 is upregulated and promotes cell proliferation in osteosarcoma. Open Med (Wars) 11: 163-167, 2016.

25. Liang S, Zhang S, Wang P, Yang C, Shang C, Yang J and Wang J: LncRNA, TUG1 regulates the oral squamous cell carcinoma progression possibly via interacting with Wnt/ $\beta$-catenin signaling. Gene 608: 49-57, 2017.

26. Xu Y, Wang J, Qiu M, Xu L, Li M, Jiang F, Yin R and Xu L: Upregulation of the long noncoding RNA TUG1 promotes proliferation and migration of esophageal squamous cell carcinoma. Tumour Biol 36: 1643-1651, 2015.

27. Huang MD, Chen WM, Qi FZ, Sun M, Xu TP, Ma P and Shu YQ: Long non-coding RNA TUG1 is up-regulated in hepatocellular carcinoma and promotes cell growth and apoptosis by epigenetically silencing of KLF2. Mol Cancer 14: 165, 2015.

28. Zeng B, Ye H, Chen J, Cheng D, Cai C, Chen G, Chen X, Xin H, Tang C and Zeng J: LncRNA TUG1 sponges miR-145 to promote cancer progression and regulate glutamine metabolism via Sirt3/GDH axis. Oncotarget 8: 113650-113661, 2017.

29. Cai H, Xue Y, Wang P, Wang Z, Li Z, Hu Y, Li Z, Shang X and Liu Y: The long noncoding RNA TUG1 regulates blood-tumor barrier permeability by targeting miR-144. Oncotarget 6: 19759-19779, 2015. 
30. Hu Y, Sun X, Mao C, Guo G, Ye S, Xu J, Zou R, Chen J, Wang L, Duan $P$ and Xue X: Upregulation of long noncoding RNA TUG1 promotes cervical cancer cell proliferation and migration. Cancer Med 6: 471-482, 2017

31. Liu L, Chen X, Zhang Y, Hu Y, Shen X and Zhu W: Long non-coding RNA TUG1 promotes endometrial cancer development via inhibiting miR-299 and miR-34a-5p. Oncotarget 8: 31386-31394, 2017.

32. Qin CF and Zhao FL: Long non-coding RNA TUG1 can promote proliferation and migration of pancreatic cancer via EMT pathway. Eur Rev Med Pharmacol Sci 21: 2377-2384, 2017.

33. Li T, Liu Y, Xiao H and Xu G: Long non-coding RNA TUG1 promotes cell proliferation and metastasis in human breast cancer. Breast Cancer 24: 535-543, 2017.

34. Iliev R, Kleinova R, Juracek J, Dolezel J, Ozanova Z, Fedorko M, Pacik D, Svoboda M, Stanik M and Slaby O: Overexpression of long non-coding RNA TUG1 predicts poor prognosis and promotes cancer cell proliferation and migration in high-grade muscle-invasive bladder cancer. Tumour Biol 37: 13385-13390, 2016.

35. Sun J, Ding C, Yang Z, Liu T, Zhang X, Zhao C and Wang J: The long non-coding RNA TUG1 indicates a poor prognosis for colorectal cancer and promotes metastasis by affecting epithelial-mesenchymal transition. J Transl Med 14: 42, 2016.

36. Niu Y, Ma F, Huang W, Fang S, Li M, Wei T and Guo L: Long non-coding RNA TUG1 is involved in cell growth and chemoresistance of small cell lung cancer by regulating LIMK2b via EZH2. Mol Cancer 16: 5, 2017.

37. Long J, Menggen Q, Wuren Q, Shi Q and Pi X: Long Noncoding RNA Taurine-Upregulated Gene1 (TUG1) promotes tumor growth and metastasis through TUG1/Mir-129-5p/Astrocyte-Elevated Gene-1 (AEG-1) axis in malignant melanoma. Med Sci Monit 24: $1547-1559,2018$

38. Lei $\mathrm{H}$, Gao Y and Xu X: LncRNA TUG1 influences papillary thyroid cancer cell proliferation, migration, and EMT formation through targeting miR-145. Acta Biochim Biophys Sin 49: 588-597, 2017

39. Ma F, Wang SH, Cai Q, Jin LY, Zhou D, Ding J and Quan ZW: Long non-coding RNA TUG1 promotes cell proliferation and metastasis by negatively regulating miR-300 in gallbladder carcinoma. Biomed Pharmacother 88: 863-869, 2017.

40. Baratieh Z, Khalaj Z, Honardoost MA, Emadi-Baygi M, Khanahmad H, Salehi M and Nikpour P: Aberrant expression of PlncRNA-1 and TUG1: Potential biomarkers for gastric cancer diagnosis and clinically monitoring cancer progression. Biomark Med 11: 1077-1090, 2017.

41. Lin PC, Huang HD, Chang CC, Chang YS, Yen JC, Lee CC, Chang WH, Liu TC and Chang JG: Long noncoding RNA TUG1, is downregulated in non-small cell lung cancer and can regulate CELF1, on binding to PRC2. BMC Cancer 16: 583, 2016.

42. Tang T, Cheng Y, She Q, Jiang Y, Chen Y, Yang W and Li Y: Long non-coding RNA TUG1 sponges miR-197 to enhance cisplatin sensitivity in triple negative breast cancer. Biomed Pharmacother 107: 338-346, 2018.

43. Li J, Zhang M, An G and Ma Q: LncRNA TUG1 acts as a tumor suppressor in human glioma by promoting cell apoptosis. Exp Biol Med (Maywood) 241: 644-649, 2016.

44. Niland CN, Merry CR and Khalil AM: Emerging roles for long non-coding RNAs in cancer and neurological disorders. Front Genet 3: 25, 2012

45. Cai H, Liu X, Zheng J, Xue Y, Ma J, Li Z, Xi Z, Li Z, Bao M and Liu Y: Long non-coding RNA taurine upregulated 1 enhances tumor-induced angiogenesis through inhibiting microRNA-299 in human glioblastoma. Oncogene 36: 318-331, 2017.

46. Katsushima K, Natsume A, Ohka F, Shinjo K, Hatanaka A, Ichimura N, Sato S, Takahashi S, Kimura H, Totoki Y, et al: Targeting the Notch-regulated non-coding RNA TUG1 for glioma treatment. Nat Commun 7: 13616, 2016.

47. Long J, Badal SS, Ye Z, Wang Y, Ayanga BA, Galvan DL, Green NH, Chang BH, Overbeek PA and Danesh FR: Long noncoding RNA Tug1 regulates mitochondrial bioenergetics in diabetic nephropathy. J Clin Invest 126: 4205-4218, 2016.

48. Kondo Y, Shinjo K and Katsushima K: Long non-coding RNAs as an epigenetic regulator in human cancers. Cancer Sci 108 : 1927-1933, 2017

49. Chiu HS, Somvanshi S, Patel E, Chen TW, Singh VP, Zorman B, Patil SL, Pan Y, Chatterjee SS; Cancer Genome Atlas Research Network, et al: Pan-cancer analysis of lncRNA regulation supports their targeting of cancer genes in each tumor context. Cell Rep 23: 297-312.e12, 2018.
50. Rinn JL, Kertesz M, Wang JK, Squazzo SL, Xu X, Brugmann SA Goodnough LH, Helms JA, Farnham PJ, Segal E and Chang HY: Functional demarcation of active and silent chromatin domains in human HOX loci by noncoding RNAs. Cell 129: 1311-1323, 2007.

51. Khalil AM, Guttman M, Huarte M, Garber M, Raj A, Morales DR, Thomas K, Presser A, Bernstein BE, van Oudenaarden A, et al: Many human large intergenic noncoding RNAs associate with chromatin-modifying complexes and affect gene expression. Proc Natl Acad Sci USA 106: 11667-11672, 2009.

52. Wahlestedt C: Targeting long non-coding RNA to therapeutically upregulate gene expression. Nat Rev Drug Discov 12: 433-446, 2013.

53. Ciferri C, Lander GC, Maiolica A, Herzog F, Aebersold R and Nogales E: Molecular architecture of human polycomb repressive complex 2. Elife 1: e00005, 2012.

54. Yoon JH, Abdelmohsen $\mathrm{K}$ and Gorospe M: Functional interactions among microRNAs and long noncoding RNAs. Semin Cell Dev Biol 34: 9-14, 2014.

55. Zhu J, Shi H, Liu H, Wang X and Li F: Long non-coding RNA TUG1 promotes cervical cancer progression by regulating the miR-138-5p-SIRT1 axis. Oncotarget 8: 65253-65264, 2017.

56. Xie Y and Zhong DW: AEG-1 is associated with hypoxia-induced hepatocellular carcinoma chemoresistance via regulating PI3K/AKT/HIF-1alpha/MDR-1 pathway. EXCLI J 15: 745-757, 2016.

57. He W, He S, Wang Z, Shen H, Fang W, Zhang Y, Qian W, Lin M, Yuan J, Wang J, et al: Astrocyte elevated gene-1(AEG-1) induces epithelial-mesenchymal transition in lung cancer through activating Wnt/ $\beta$-catenin signaling. BMC Cancer 15: 107, 2015.

58. Yan G, Wang X, Yang M, Lu L and Zhou Q: Long non-coding RNA TUG1 promotes progression of oral squamous cell carcinoma through upregulating FMNL2 by sponging miR-219. Am J Cancer Res 7: 1899-1912, 2017.

59. Zhao XB and Ren GS: LncRNA taurine-upregulated gene 1 promotes cell proliferation by inhibiting MicroRNA-9 in MCF-7 cells. J Breast Cancer 19: 349-357, 2016.

60. Yang B, Tang X, Wang Z, Sun D, Wei X and Ding Y: TUG1 promotes prostate cancer progression by acting as a ceRNA of miR-26a. Biosci Rep 38: pii: BSR20180677, 2018,

61. Zhao L, Sun H, Kong H, Chen Z, Chen B and Zhou M: The Lncrna-TUG1/EZH2 axis promotes pancreatic cancer cell proliferation, migration and EMT phenotype formation through sponging Mir-382. Cell Physiol Biochem 42: 2145-2158, 2017.

62. Guo P, Zhang G, Meng J, He Q, Li Z and Guan Y: Upregulation of long non-coding RNA TUG1 promotes bladder cancer cell proliferation, migration, and invasion by inhibiting miR-29c. Oncol Res 26: 1083-1091, 2018.

63. Liu Q, Liu H, Cheng H, Li Y, Li X and Zhu C: Downregulation of long noncoding RNA TUG1 inhibits proliferation and induces apoptosis through the TUG1/miR-142/ZEB2 axis in bladder cancer cells. Onco Targets Ther 10: 2461-2471, 2017.

64. Tan J, Qiu K, Li M and Liang Y: Double-negative feedback loop between long non-coding RNA TUG1 and miR-145 promotes epithelial to mesenchymal transition and radioresistance in human bladder cancer cells. FEBS Lett 589: 3175-3181, 2015.

65. Li J, Zhang Q, Fan X, Mo W, Dai W, Feng J, Wu L, Liu T, Li S, Xu S, et al: The long noncoding RNA TUG1 acts as a competing endogenous RNA to regulate the Hedgehog pathway by targeting miR-132 in hepatocellular carcinoma. Oncotarget 8 : 65932-65945, 2017.

66. Bakshi A, Chaudhary SC, Rana M, Elmets CA and Athar M: Basal cell carcinoma pathogenesis and therapy involving hedgehog signaling and beyond. Mol Carcinog 56: 2543-2557, 2017 .

67. Lv J, Kong Y, Gao Z, Liu Y, Zhu P and Yu Z: LncRNA TUG interacting with miR-144 contributes to proliferation, migration and tumorigenesis through activating the JAK2/STAT3 pathway in hepatocellular carcinoma. Int J Biochem Cell Biol 101: 19-28, 2018.

68. Xie C, Chen B, Wu B, Guo J and Cao Y: LncRNA TUG1 promotes cell proliferation and suppresses apoptosis in osteosarcoma by regulating miR-212-3p/FOXA1 axis. Biomed Pharmacother 97: $1645-1653,2018$

69. Li G, Liu K and Du X: Long non-coding RNA TUG1 promotes proliferation and inhibits apoptosis of osteosarcoma cells by sponging miR-132-3p and Upregulating SOX4 expression. Yonsei Med J 59: 226-235, 2018.

70. Cao J, Han X, Qi X, Jin X and Li X: TUG1 promotes osteosarcoma tumorigenesis by upregulating EZH2 expression via miR-144-3p. Int J Oncol 51: 1115-1123, 2017. 
71. Wang H, Yu Y, Fan S and Luo L: Knockdown of long noncoding RNA TUG1 inhibits the proliferation and cellular invasion of osteosarcoma cells by sponging MiR-153. Oncol Res 26 665-673, 2018

72. Xie CH, Cao YM, Huang Y, Shi QW, Guo JH, Fan ZW, Li JG, Chen BW and Wu BY: Long non-coding RNA TUG1 contributes to tumorigenesis of human osteosarcoma by sponging miR-9-5p and regulating POU2F1 expression. Tumour Biol 37: 15031-15041, 2016.

73. Li Y, Zhang T, Zhang Y, Zhao X and Wang W: Targeting the FOXM1-regulated long non-coding RNA TUG1 in osteosarcoma. Cancer Sci 109: 3093-3104, 2018.

74. Zhang EB, Yin DD, Sun M, Kong R, Liu XH, You LH, Han L, Xia R, Wang KM, Yang JS, et al: P53-regulated long non-coding RNA TUG1 affects cell proliferation in human non-small cell lung cancer, partly through epigenetically regulating HOXB7 expression. Cell Death Dis 5: e1243, 2014.

75. Liao WT, Jiang D, Yuan J, Cui YM, Shi XW, Chen CM, Bian XW, Deng YJ and Ding YQ: HOXB7 as a prognostic factor and mediator of colorectal cancer progression. Clin Cancer Res 17: 3569-3578, 2011

76. Zhang E, He X, Yin D, Han L, Qiu M, Xu T, Xia R, Xu L, Yin R and De W: Increased expression of long noncoding RNA TUG1 predicts a poor prognosis of gastric cancer and regulates cell proliferation by epigenetically silencing of p57. Cell Death Dis 7: e2109, 2016.

77. Shen P, Sun J, Xu G, Zhang L, Yang Z, Xia S, Wang Y, Liu Y and Shi G: KLF9, a transcription factor induced in flutamide-caused cell apoptosis, inhibits AKT activation and suppresses tumor growth of prostate cancer cells. Prostate 74: 946-958, 2014.

78. Liu H, Zhou G, Fu X, Cui H, Pu G, Xiao Y, Sun W, Dong X, Zhang L, Cao S, et al: Long noncoding RNA TUG1 is a diagnostic factor in lung adenocarcinoma and suppresses apoptosis via epigenetic silencing of BAX. Oncotarget 8: 101899-101910, 2017.

79. Li T, Chen Y, Zhang J and Liu S: LncRNA TUG1 promotes cells proliferation and inhibits cells apoptosis through regulating AURKA in epithelial ovarian cancer cells. Med (Baltimore) 97: e12131, 2018

80. Yang LY, He CY, Chen XH, Su LP, Liu BY and Zhang H: Aurora kinase A revives dormant laryngeal squamous cell carcinoma cells via FAK/PI3K/Akt pathway activation. Oncotarget 7: 48346-48359, 2016.

81. Schnepp RW, Khurana P, Attiyeh EF, Raman P, Chodosh SE, Oldridge DA, Gagliardi ME, Conkrite KL, Asgharzadeh S Seeger RC, et al: A LIN28B-RAN-AURKA signaling network promotes neuroblastoma tumorigenesis. Cancer Cell 28: 599-609, 2015

82. Wang L, Zhao Z, Feng W, Ye Z, Dai W, Zhang C, Peng J and Wu K: Long non-coding RNA TUG1 promotes colorectal cancer metastasis via EMT pathway. Oncotarget 7: 51713-51719, 2016.

83. Yin DD, Zhang EB, You LH, Wang N, Wang LT, Jin FY, Zhu YN Cao LH, Yuan QX, De W and Tang W: Downregulation of lncRNA TUG1 affects apoptosis and insulin secretion in mouse pancreatic $\beta$ cells. Cell Physiol Biochem 35: 1892-1904, 2015.

84. Yilmaz M and Christofori G: EMT, the cytoskeleton, and cancer cell invasion. Cancer Metastasis Rev 28: 15-33, 2009.

85. Vergara D, Merlot B, Lucot JP, Collinet P, Vinatier D, Fournier I and Salzet M: Epithelial-mesenchymal transition in ovarian cancer. Cancer Lett 291: 59-66, 2010.
86. Sun JF, Hu JY, Wang GJ, Yang Z, Zhao C, Zhang X and Wang J: LncRNA TUG1 promoted KIAA1199 expression via miR-600 to accelerate cell metastasis and epithelial-mesenchymal transition in colorectal cancer. J Exp Clin Cancer Res 37: 106, 2018.

87. Wang Y, Yang T, Zhang Z, Lu M, Zhao W, Zeng X and Zhang W: Long non-coding RNA TUG1 promotes migration and invasion by acting as a ceRNA of miR-335-5p in osteosarcoma cells. Cancer Sci 108: 859-867, 2017.

88. Ji TT, Huang X, Jin J, Pan SH and Zhuge XJ: Inhibition of long non-coding RNA TUG1 on gastric cancer cell transference and invasion through regulating and controlling the expression of miR-144/c-Met axis. Asian Pac J Trop Med 9: 508-512, 2016.

89. He C, Liu Z, Jin L, Zhang F, Peng X, Xiao Y, Wang X, Lyu Q and Cai X: lncRNA TUG1-mediated Mir-142-3p downregulation contributes to metastasis and the Epithelial-to-Mesenchymal transition of hepatocellular carcinoma by targeting ZEB1. Cell Physiol Biochem 48: 1928-1941, 2018.

90. Lin YH, Wu MH, Huang YH, Yeh CT, Cheng ML, Chi HC, Tsai CY, Chung IH, Chen CY and Lin KH: Taurine up-regulated gene 1 functions as a master regulator to coordinate glycolysis and metastasis in hepatocellular carcinoma. Hepatology 67: 188-203, 2018

91. Li C, Gao Y, Li Y and Ding D: TUG1 mediates methotrexate resistance in colorectal cancer via miR-186/CPEB2 axis. Biochem Biophys Res Commun 491: 552-557, 2017.

92. Shafiee SM, Rasti M, Seghatoleslam A, Azimi T and Owji AA UBE2Q1 in a human breast carcinoma cell line: Overexpression and interaction with p53. Asian Pac J Cancer Prev 16: 3723-3727, 2015.

93. Lee K, Jung ES, Choi YJ, Lee KY and Lee A: Expression of pRb, p53, p16 and cyclin D1 and their clinical implications in urothelial carcinoma. J Korean Med Sci 25: 1449-1455, 2010.

94. Jiang L, Zhang Q, Ren H, Ma S, Lu C, Liu B, Liu J, Liang J, Li M and Zhu R: Dihydromyricetin enhances the chemo-sensitivity of nedaplatin via regulation of the $\mathrm{p} 53 / \mathrm{Bcl}-2$ pathway in hepatocellular carcinoma cells. PLoS One 10: e0124994, 2015.

95. Elhag R, Mazzio EA and Soliman KF: The effect of silibinin in enhancing toxicity of temozolomide and etoposide in p53 and PTEN-mutated resistant glioma cell lines. Anticancer Res 35: 1263-1269, 2015.

96. Pilarsky C, Wenzig M, Specht T, Saeger HD and Grützmann R: Identification and validation of commonly overexpressed genes in solid tumors by comparison of microarray data. Neoplasia 6 : 744-750, 2004.

97. Okabe H, Satoh S, Kato T, Kitahara O, Yanagawa R, Yamaoka Y, Tsunoda T, Furukawa Y and Nakamura Y: Genome-wide analysis of gene expression in human hepatocellular carcinomas using cDNA microarray: Identification of genes involved in viral carcinogenesis and tumor progression. Cancer Res 61: 2129-2137, 2001.

98. Li J, Zhang M, An G and Ma Q: Long non-coding RNA TUG1 acts as a miR-26a sponge in human glioma cells. Biochem Biophys Res Commun 477: 743-748, 2016.

This work is licensed under a Creative Commons Attribution-NonCommercial-NoDerivatives 4.0 International (CC BY-NC-ND 4.0) License. 